\title{
Ethik, Evidenz und Eigeninteresse: Für eine offene Diskurskultur
}

\author{
Georg Marckmann · Gerald Neitzke
}

Online publiziert: 16 . Oktober 2015

(C) Springer-Verlag Berlin Heidelberg 2015

Im März diesen Jahres publizierten Hartmut Bettin, Alexander Spassov und Micha H. Werner online in dieser Zeitschrift eine ethische Analyse, die sich kritisch mit dem Bedarf und der Legitimität kieferorthopädischer Behandlungen auseinandersetzte [2]. Einem Artikel in Spiegel Online (10.06.2015) zufolge wurde die Veröffentlichung von der Präsidentin der Deutschen Gesellschaft für Kieferorthopädie kritisiert [4]. Diese Kritik wurde jedoch nicht als Kommentar oder Leserbrief in der Zeitschrift Ethik in der Medizin geäußert, sondern gegenüber dem Vorgesetzten eines der Autoren. In der Folge wurde der betreffende Autor laut Spiegel Online angewiesen, alle Veröffentlichungen vorab dem Direktor der Klinik vorzulegen, zudem wurde ein Zusammenhang mit der Nichtverlängerung seines Arbeitsvertrags unterstellt. Inwieweit sich diese Vorkommnisse tatsächlich in der berichteten Art und Weise zugetragen haben, können und wollen wir in dem vorliegenden Editorial nicht beurteilen. Vielmehr möchten wir die Kontroverse zum Anlass nehmen, den Stellenwert ethischer Analysen im Spannungsfeld zwischen der wissenschaftlichen Selbstreflexion professionellen Handelns einerseits und fachlichen Eigeninteressen andererseits zu reflektieren.

Es gehört zu den zentralen Aufgaben der Medizinethik, ethische Fragen in der Gesundheitsversorgung zu identifizieren, zu analysieren und mit einer Bewertung der Argumente zu einer begründeten Entscheidungsfindung beizutragen. Dabei geht es nicht nur um die klassischen ethisch-dilemmatischen Entscheidungssituationen im Einzelfall, sondern auch um grundlegende Fragen der Legitimation ärztlichen und pflegerischen Handelns. Diese

Univ.-Prof. Dr. med. G. Marckmann, MPH ( $₫)$

Institut für Ethik, Geschichte und Theorie der Medizin,

Ludwig-Maximilians-Universität München,

Lessingstr. 2,

80336 München, Deutschland

E-Mail: marckmann@lmu.de

Dr. med. G. Neitzke

Institut für Geschichte, Ethik und Philosophie der Medizin,

Medizinische Hochschule Hannover,

Hannover, Deutschland 
ergibt sich häufig nicht allein aus dem medizinischen oder pflegerischen Fachwissen, sondern setzt normative Urteile voraus, die auf individuelle und gesellschaftliche Wertvorstellungen verweisen. Die Frage nach dem Sinn einer Behandlungsmaßnahme lässt sich nicht ohne Rückgriff auf das Menschenbild und das Wissenschaftsverständnis klären. Wann ist ein bestimmter Gesundheitszustand behandlungsbedürftig und legitimiert damit ein ärztliches Eingreifen? Wie sind die Nutzen- und Schadenspotenziale der verfügbaren medizinischen Maßnahmen einzuschätzen und ggf. gegeneinander abzuwägen? Genau diesen Fragen widmet sich der Artikel von Bettin et al. im Bereich der kieferorthopädischen Praxis: Welche Gründe liegen kieferorthopädischen Behandlungen zugrunde? Welche Erwartungen haben diejenigen, die die Behandlungsleistungen in Anspruch nehmen? In welchem Verhältnis stehen Aspekte der oralen Gesundheit und ästhetische Gesichtspunkte? Und: Welche Kriterien liegen der Kostenübernahme durch die Krankenkassen zugrunde? Zudem prüfen die Autoren die verfügbare wissenschaftliche Evidenz zu den erwartbaren Funktionseinschränkungen durch Zahnstellungsvariationen, da sie eine tragende Säule der Legitimierung der Behandlung darstellt. Die Untersuchung der Legitimität kieferorthopädischer Behandlungen wirft damit fachliche, individualethische und sozial- bzw. gerechtigkeitsethische Fragen auf.

Die Autoren, die sowohl über fachwissenschaftliche als auch philosophisch-ethische Kompetenz verfügen, kommen zu dem Schluss, dass die Antworten auf die verschiedenen Fragen bislang ,nicht zwanglos zu vereinbaren sind“. Während eine Mehrheit der Bevölkerung den Zweck kieferorthopädischer Behandlungen in der Verbesserung des Erscheinungsbildes sieht, legitimieren die Kieferorthopäden ihre Behandlung mit einer Gefährdung der oralen Gesundheit und der Funktionsfähigkeit des Gebisses. Eine ähnliche Rechtfertigung liegt der Kostenübernahme durch die Krankenkassen zugrunde: Die Behandlung muss in der Lage sein, eine aktuelle oder drohende erhebliche Funktionsbeeinträchtigung erfolgreich zu beheben. Nach Einschätzung der Autoren ist der Zusammenhang zwischen „Kiefer- oder Zahnfehlstellungen“ und späteren Funktionseinschränkungen wissenschaftlich unzureichend belegt, sodass der Nachweis einer ,Zahnstellungsanomalie“ allein keine hinreichende Rechtfertigung für eine kieferorthopädische Behandlung darstellt - sofern die (erwartete) Funktionseinschränkung das für die Behandlungsbedürftigkeit entscheidende normative Kriterium ist.

Es entspricht guter wissenschaftlicher Praxis in der Medizinethik, dass die Autoren die ethischen Fragen nicht nur identifizieren und analysieren, sondern auch prüfen, welche Lösungen für die Problemkonstellationen angemessen sein könnten. Sie zeigen vier Möglichkeiten auf und weisen zu Recht darauf hin, dass nur im interdisziplinären Diskurs entschieden werden kann, welcher Option der Vorrang einzuräumen ist. Obgleich sich die Autoren damit der begrenzten Reichweite ihrer wissenschaftlich-ethischen Analyse bewusst sind, besitzen die Ergebnisse allein durch die Infragestellung der Indikation kieferorthopädischer Behandlungen - potenziell - eine erhebliche Tragweite. Zum einen berühren sie das professionelle Urteil der praktisch tätigen Kieferorthopäden über die Angemessenheit ihrer Behandlungen. Zum anderen haben diese Urteile wiederum eine erhebliche ökonomische Bedeutung: als Verdienstmöglichkeit für die Behandler und als Ausgabenfaktor für die Kostenträger. Es überrascht deshalb wenig, dass der Beitrag von Bettin et al. Reaktionen der betroffenen Interessengruppen provoziert. 
Dass (Zahn-)Ärzte mit ihren Behandlungen Geld verdienen, ist nicht moralisch verwerflich. Allerdings resultieren Interessenkonflikte auf unterschiedlichen Ebenen, die zu (betriebs-)wirtschaftlich motivierter Über- oder Unterversorgung führen können. Dies schadet den Patienten und stellt - im Falle der Überversorgung - eine Verschwendung gemeinschaftlich aufgebrachter, begrenzt verfügbarer Ressourcen dar. In der Verantwortung sind dabei nicht nur die einzelnen Leistungserbringer, die mit ihrer Indikationsstellung im Einzelfall wesentlich den Mittelverbrauch steuern, sondern auch die wissenschaftlichen medizinischen Fachgesellschaften, die z. B. über die Erstellung klinischer Leitlinien ebenfalls Einfluss auf den Einsatz medizinischer Maßnahmen ausüben können. Es muss folglich als eine wesentliche Aufgabe der Fachgesellschaften angesehen werden, sich an Diskursen über die Angemessenheit von Indikationen, wie sie z. B. durch den Beitrag von Bettin et al. für den Bereich der Kieferorthopädie angeregt wurden, zu beteiligen. Als wissenschaftiche Fachgesellschaften sollten sie dies aber mit Bezug auf die beste verfügbare Evidenz auf den üblichen Wegen tun. Im vorliegenden Fall hätte dies eine evidenzbasierte wissenschaftliche Publikation zur Indikationsstellung bei kieferorthopädischen Behandlungen sein können, aber auch ein Kommentar oder Leserbrief in dieser Zeitschrift. Ein solcher Kommentar wäre als Beitrag zur Qualitätssicherung der vorgenommenen ethischen Analysen willkommen.

Dass dabei auch auf der Ebene medizinisch-wissenschaftlicher Fachgesellschaften Interessenkonflikte resultieren können, liegt auf Hand, da ihre Mitglieder in der Regel nicht nur forschen, sondern gleichzeitig als Leistungserbringer finanziell vom Einsatz der Behandlungsverfahren profitieren. Die Arbeitsgemeinschaft der Wissenschaftlichen Medizinischen Fachgesellschaften (AWMF) hat die Frage der Interessenkonflikte bei Fachgesellschaften bereits 2010 in einer Empfehlung aufgegriffen, fokussiert dabei aber vor allem diejenigen Interessenkonflikte, die durch finanzielle Zuwendungen der Industrie bei Publikationen, Kongressen und der Erarbeitung von Leitlinien entstehen [1]. Eine darüber hinausgehende Reflexion möglicher Konflikte mit den eigenen wirtschaftlichen Interessen erschiene durchaus wünschenswert. Vom Grundsatz her zu begrüßen ist in diesem Zusammenhang die vom American Board of Internal Medicine (ABIM) begründete Choosing Wisely Initiative (www.choosingwisely.org, zugegriffen: 06. Okt. 2015), bei der verschiedene Fachgesellschaften sich auf jeweils fünf Maßnahmen verständigen, die bei bestimmten Erkrankungen unterlassen werden sollten, um Überversorgung zu reduzieren [3]. Unter dem Motto „Gemeinsam Klug Entscheiden“ hat die AWMF das Thema aufgegriffen und Kriterien für die Qualität der Empfehlungen formuliert; die Deutsche Gesellschaft für Innere Medizin (DGIM) möchte mit der Initiative „Klug entscheiden“ bis zum Ende des Jahres 2015 nicht nur die Top 5 der Überversorgung, sondern auch der Unterversorgung in elf Subspezialisierungen der Inneren Medizin identifizieren. Mit diesen Initiativen können die Fachgesellschaften einen wesentlichen Beitrag leisten zur kritischen Reflexion professioneller Urteile über den sinnvollen Einsatz der verfügbaren diagnostischen und therapeutischen Verfahren. Sofern dabei, wie in der Kieferorthopädie, notwendig Werturteile erforderlich sind, sollten die Fachgesellschaften den Austausch mit anderen Disziplinen wie z. B. der Ethik in der Medizin und den öffentlichen Diskurs suchen. Zur (selbst-)kritischen Kultur wissenschaftlicher Fachgesellschaften gehört zweifellos auch, dass ihre Mitglieder die Grundlagen professioneller Entscheidungen wissenschaftlich reflektieren können, ohne dadurch persönliche Nachteile befürchten zu müssen. Von einem offenen, selbstkritischen Diskurs innerhalb der Fächer und Fachgesellschaften werden am Ende diejenigen profitieren, deren Interessen im Mittelpunkt stehen müssen: die Patientinnen und Patienten. 


\section{Literatur}

1. Arbeitsgemeinschaft der Wissenschaftlichen Medizinischen Fachgesellschaften (2010) Empfehlungen der AWMF zum Umgang mit Interessenkonflikten bei Fachgesellschaften. Düsseldorf. http://www. awmf.org/fileadmin/user_upload/Leitlinien/Werkzeuge/empf-coi.pdf. Zugegriffen: 1. Okt. 2015

2. Bettin H, Spassov A, Werner MH (2015) Asymmetrien bei der Einschätzung des kieferorthopädischen Behandlungsbedarfs. Kieferorthopädische Praxis und kieferorthopädische Normen im Spannungsfeld der Interessen von Patient, Arzt und Gesellschaft. Ethik Med 27:183-196

3. Strech D, Follmann M, Klemperer D, Lelgemann M, Ollenschlager G, Raspe H et al (2014) When choosing wisely meets clinical practice guidelines. Z Evid Fortbild Qual Gesundhwes 108(10):601-603

4. Wolf T (2015) Kieferorthopädie: Kritik an Zahnspange unerwünscht. Spiegel online 10.06.15. http://www.spiegel.de/gesundheit/diagnose/zahnspange-kritik-an-kieferorthopaedie-unerwuenscht-a-1037018.html. Zugegriffen: 1. Okt. 2015 